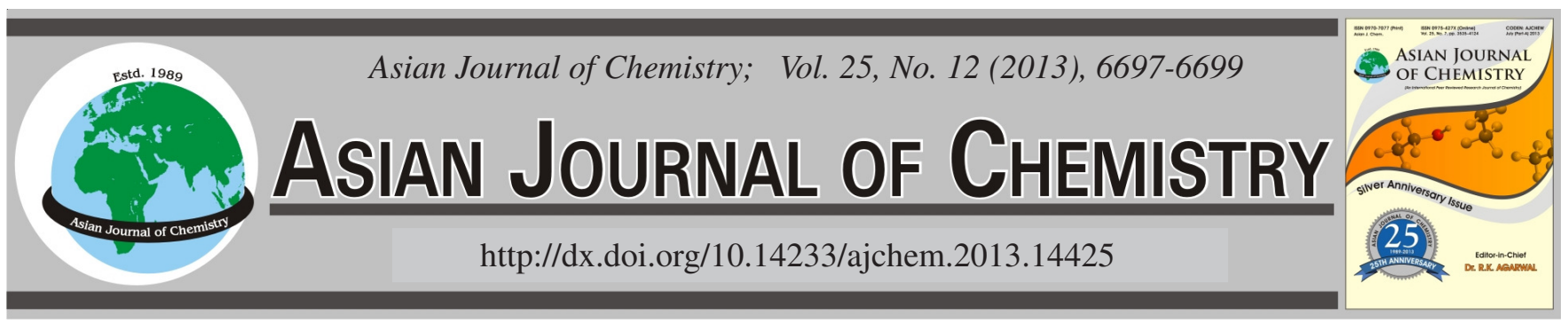

\title{
Synthesis and Biological Activities of 3-(2-Chloro-3,3,3-trifluoroprop-1-enyl)- 2,2-dimethyl-N-Substituted Phenylcyclopropanecarboxamide
}

\author{
Min-Jun Wang, Na-Bo Sun ${ }^{*}$, Jian-Zhong Jin, Jian-Fen Zhang and Hui-Ying Xu
}

College of Biology and Environmental Engineering, Zhejiang Shuren University, Hangzhou 310015, P.R. China

*Corresponding author: E-mail: nabosun@gmail.com

Some amide compounds containing pyrethroids are synthesized. Their structures were confirmed by ${ }^{1} \mathrm{H}$ NMR, MS and elemental analysis. The bioassay results indicated that they showed moderate fungicidal activity.

Key Words: Cyclopropanecarboxamide, Synthesis, Fungicidal activity.

\section{INTRODUCTION}

Cyclopropane derivatives, as a kind of highly bioactive compounds, have been studied broadly for many years ${ }^{1}$. In 1940s, cyclopropane compounds, especially pyrethroids were marketed as low toxic insecticides. From then, a large variety of pyrethroids derivatives have been synthesized and lots of them, such as deltamethrin, cypermethrin, bifenthrin, fenvalerate, tefluthrin, etc. are commercially available ${ }^{2}$. So it is a research hotspot in agriculture, many biologically active and structurally stable cyclopropane compounds had been synthesized $^{3}$.

Amide group is widely used in medicinal chemistry and agricultural chemistry, because of their excellent biological activities ${ }^{4}$. It displayed broad biological activities, such as herbicidal, fungicidal and insecticidal activities, etc. Due to their variety bioactivities and in line with our continuous efforts to synthesize bioactive compounds for crop protection, 3-(2chloro-3,3,3-trifluoroprop-1-enyl)-2,2-dimethyl-N-substituted phenylcyclopropanecarboxamide were designed some amide compounds and their biological activity tested.

\section{EXPERIMENTAL}

Melting points were determined by an X-4 apparatus and uncorrected. ${ }^{1} \mathrm{H}$ NMR spectra were measured on a Bruker Avance 400 DMX instrument using TMS as an internal standard and $\mathrm{CDCl}_{3}$ as the solvent. Mass spectra were recorded on a HP 5989B mass detector instrument. Elemental analyses were performed on a Carlo erba EA1110 elemental analyzer. All the reagents are of analytical grade or freshly prepared before use.
General procedure: $\lambda$-Cyhalthrin acid was synthesized in our laboratory according to literature. Thionyl chloride $(100 \mathrm{~mL})$ was added into lambda cyhalthrin acid $(2.75 \mathrm{~g}, 10$ $\mathrm{mmol}$ ) and the mixture was refluxed for $8 \mathrm{~h}$. Next, the excessive thionyl chloride was distilled off under reduced pressure. The desired acid chloride was not purification. Dropwised the acid chloride to substituted aniline $(10 \mathrm{mmol})$ and $\mathrm{Et}_{3} \mathrm{~N}(12 \mathrm{mmol})$, then vigorously stirred at ambient temperature. TLC was employed to trace the process. Stop the reaction and cool the resultant mixture under room temperature. The corresponding amide precipitated immediately. The product was filtered, washed with diluted acetic acid, dried and purified by silicagel column eluted with ethyl acetate/petroleum ether to give the title compounds 4 .

3-(2-Chloro-3,3,3-trifluoroprop-1-enyl)-2,2-dimethylN-phenylcyclopropanecarboxamide (4a): White crystal; yield, $82.3 \%$; m.p. $143-146{ }^{\circ} \mathrm{C} ;{ }^{1} \mathrm{H}$ NMR $\left(\mathrm{CDCl}_{3}\right) \delta: 1.33$ (s, $\left.3 \mathrm{H}, \mathrm{CH}_{3}\right), 1.38\left(\mathrm{~s}, 3 \mathrm{H}, \mathrm{CH}_{3}\right), 1.82(\mathrm{~d}, J=8.4 \mathrm{~Hz}, 1 \mathrm{H}$, cyclopropane $\mathrm{H}), 2.19(\mathrm{t}, J=8.8 \mathrm{~Hz}, 1 \mathrm{H}$, cyclopropane $\mathrm{H}), 7.09$ $7.13(\mathrm{~m}, 1 \mathrm{H}, \mathrm{Ph}), 7.17$ (d, J = 9.2 Hz, 1H, C=CH), 7.30-7.36 (m, 2H, Ph), 7.46-7.48 (m, 2H, Ph), 7.80 (s, 1H, NH); Ms m/ z (relative intensity/\%): $317\left(\mathrm{M}^{+}, 42\right), 282(63), 224$ (62), 198 (55), 141 (17), 120 (90), 93 (20), 77 (11). Anal. calcd. (\%) for $\mathrm{C}_{15} \mathrm{H}_{15} \mathrm{NOClF}_{3}$ : C 56.70, H 4.76, N 4.41, found (\%): C 56.82, $\mathrm{H} 4.80, \mathrm{~N} 4.50$.

3-(2-Chloro-3,3,3-trifluoroprop-1-enyl)-2,2-dimethylN-(4-chlorophenyl)cyclopropanecarboxamide (4b): White crystal; yield, $78.2 \%$; m.p. $143-145{ }^{\circ} \mathrm{C} ;{ }^{1} \mathrm{H}$ NMR $\left(\mathrm{CDCl}_{3}\right) \delta$ : 1.33 (s, 3H, $\left.\mathrm{CH}_{3}\right), 1.37$ (s, 3H, $\left.\mathrm{CH}_{3}\right), 1.80$ (d, J=8.0 Hz, $1 \mathrm{H}$, cyclopropane $\mathrm{H}), 2.20$ (t, $J=8.8 \mathrm{~Hz}, 1 \mathrm{H}$, cyclopropane $\mathrm{H}$ ), $7.13(\mathrm{~d}, J=9.6 \mathrm{~Hz}, 1 \mathrm{H}, \mathrm{C}=\mathrm{CH}), 7.26-7.33$ (m, 2H, Ph), 7.42- 
$7.44(\mathrm{~m}, 2 \mathrm{H}, \mathrm{Ph}), 7.81$ (s, 1H, NH); Ms m/z (relative intensity/ \%): $352\left(\mathrm{M}^{+}, 27\right), 316$ (19), 258 (25), 225 (19), 198 (43), 163 (30), 154 (100), 141 (42), 126 (78), 99 (19), 91 (13), 75 (17); Anal. calcd. (\%) for $\mathrm{C}_{15} \mathrm{H}_{14} \mathrm{NOCl}_{2} \mathrm{~F}_{3}$ : C 51.16, H 4.01, N 3.98, found (\%): C 51.22, H 4.05, N 3.86.

3-(2-Chloro-3,3,3-trifluoroprop-1-enyl)-2,2-dimethylN-(2-chlorophenyl)cyclopropanecarboxamide (4c): White crystal; yield, $80.7 \%$; m.p. $128-131{ }^{\circ} \mathrm{C} ;{ }^{1} \mathrm{H} \mathrm{NMR}\left(\mathrm{CDCl}_{3}\right) \delta$ : $1.36\left(\mathrm{~s}, 3 \mathrm{H}, \mathrm{CH}_{3}\right), 1.38$ (s, 3H, $\left.\mathrm{CH}_{3}\right), 1.90$ (d, $J=8.0 \mathrm{~Hz}, 1 \mathrm{H}$, cyclopropane $\mathrm{H}), 2.23(\mathrm{t}, J=8.8 \mathrm{~Hz}, 1 \mathrm{H}$, cyclopropane $\mathrm{H})$, 7.02-7.06 (m, 1H, Ph), 7.12 (d, J=9.2 Hz, 1H, C=CH), 7.247.28 (m, 2H, Ph), 7.36-7.38 (m, 1H, Ph), 7.77(s, 1H, NH); Ms $\mathrm{m} / \mathrm{z}$ (relative intensity/\%): $352\left(\mathrm{M}^{+}, 57\right), 316(23), 258(24)$, 225 (19), 198 (41), 163 (22), 154 (100), 141 (37), 126 (72), 99(19), 91(13), 75(10); anal. calcd. (\%) for $\mathrm{C}_{15} \mathrm{H}_{14} \mathrm{NOCl}_{2} \mathrm{~F}_{3}$ : C 51.16, H 4.01, N 3.98, found (\%): C 51.25, H 4.04, N 3.93.

3-(2-Chloro-3,3,3-trifluoroprop-1-enyl)-2,2-dimethylN-(3-phenyl)cyclopropanecarboxamide (4d): White crystal; yield, $76.8 \%$; m.p. $129-131{ }^{\circ} \mathrm{C} ;{ }^{1} \mathrm{H}$ NMR $\left(\mathrm{CDCl}_{3}\right) \delta: 1.33(\mathrm{~s}$, $\left.3 \mathrm{H}, \mathrm{CH}_{3}\right), 1.37$ (s, 3H, $\left.\mathrm{CH}_{3}\right), 1.79$ (d, $J=8.0 \mathrm{~Hz}, 1 \mathrm{H}$, cyclopropane $\mathrm{H}), 2.19$ (t, $J=8.8 \mathrm{~Hz}, 1 \mathrm{H}$, cyclopropane $\mathrm{H}), 7.08$ (d, $J=7.2 \mathrm{~Hz}, 1 \mathrm{H}, \mathrm{C}=\mathrm{CH}), 7.11-7.34(\mathrm{~m}, 4 \mathrm{H}, \mathrm{Ph}), 7.60(\mathrm{~s}, 1 \mathrm{H}$, $\mathrm{NH}) ; \mathrm{Ms} \mathrm{m} / \mathrm{z}$ (relative intensity/\%): $352\left(\mathrm{M}^{+}, 55\right), 316(30)$, 258 (28), 225 (25), 198 (59), 163 (38), 154 (100), 141 (49), 126 (73), 111 (46), 91 (16), 75 (19); anal. calcd. (\%) for $\mathrm{C}_{15} \mathrm{H}_{14} \mathrm{NOCl}_{2} \mathrm{~F}_{3}$ : C 51.16, H 4.01, N 3.98, found (\%): C 51.27, $\mathrm{H} 3.97$, N 4.05.

3-(2-Chloro-3,3,3-trifluoroprop-1-enyl)-2,2-dimethylN-(4-methylphenyl)cyclopropanecarboxamide (4e): White crystal; yield, 83.1 \%; m.p. $129-130{ }^{\circ} \mathrm{C}$; ${ }^{1} \mathrm{H}$ NMR $\left(\mathrm{CDCl}_{3}\right) \delta$ : 1.32 (s, 3H, $\left.\mathrm{CH}_{3}\right), 1.36$ (s, 3H, $\left.\mathrm{CH}_{3}\right), 1.80$ (d, J = 8.0 Hz, $1 \mathrm{H}$, cyclopropane $\mathrm{H}), 2.16(\mathrm{t}, J=8.8 \mathrm{~Hz}, 1 \mathrm{H}$, cyclopropane $\mathrm{H})$, $2.30\left(\mathrm{~s}, 3 \mathrm{H}, \mathrm{CH}_{3}\right), 7.11(\mathrm{~d}, J=8.0 \mathrm{~Hz}, 1 \mathrm{H}, \mathrm{C}=\mathrm{CH}), 7.16-7.18$ (m, 2H, Ph), 7.32-7.34 (m, 2H, Ph), 7.62 (s, 1H, NH); Ms m/ $\mathrm{z}$ (relative intensity/\%): $332\left(\mathrm{M}^{+}, 31\right), 296$ (23), 238 (19), 225 (7), 198 (10), 134 (74), 106 (100), 91 (26), 77 (23); anal. calcd. (\%) for $\mathrm{C}_{16} \mathrm{H}_{17} \mathrm{NOClF}_{3}$ : C 57.92, $\mathrm{H} 5.16, \mathrm{~N} 4.22$, found (\%): C $58.03, \mathrm{H} 5.12, \mathrm{~N} 4.16$.

3-(2-Chloro-3,3,3-trifluoroprop-1-enyl)-2,2-dimethylN-(2-methylphenyl)cyclopropanecarboxamide (4f): White crystal; yield, $81.3 \%$; m.p. $140-143{ }^{\circ} \mathrm{C}$; ${ }^{1} \mathrm{H} \mathrm{NMR}\left(\mathrm{CDCl}_{3}\right) \delta$ : 1.35 (s, 3H, $\left.\mathrm{CH}_{3}\right), 1.38$ (s, 3H, $\left.\mathrm{CH}_{3}\right), 1.87$ (d, $J=8.0 \mathrm{~Hz}, 1 \mathrm{H}$, cyclopropane $\mathrm{H}), 2.20(\mathrm{t}, J=8.8 \mathrm{~Hz}, 1 \mathrm{H}$, cyclopropane $\mathrm{H})$, $2.26\left(\mathrm{~s}, 3 \mathrm{H}, \mathrm{CH}_{3}\right), 7.09(\mathrm{~d}, J=7.2 \mathrm{~Hz}, 1 \mathrm{H}, \mathrm{C}=\mathrm{CH}), 7.15-7.25$ (m, 4H, Ph), $7.70(\mathrm{~s}, 1 \mathrm{H}, \mathrm{NH}) ; \mathrm{Ms} \mathrm{m} / \mathrm{z}$ (relative intensity/\%): $332\left(\mathrm{M}^{+}, 44\right), 296(26), 238(11), 225(12), 198(14), 134$ (100), 106(79), 91(50), 77(23). $\mathrm{C}_{16} \mathrm{H}_{17} \mathrm{NOClF}_{3}$; anal. calcd. (\%) for $\mathrm{C}_{16} \mathrm{H}_{17} \mathrm{NOClF}_{3}$ : C 58.92, H 5.16, N 4.22, found (\%): C 60.06, H 5.12, N 4.13.

3-(2-Chloro-3,3,3-trifluoroprop-1-enyl)-2,2-dimethylN-(3-methylphenyl)cyclopropanecarboxamide (4g): White crystal; yield, $86.8 \%$; m.p. $137-139{ }^{\circ} \mathrm{C} ;{ }^{1} \mathrm{H} \mathrm{NMR}\left(\mathrm{CDCl}_{3}\right) \delta$ : $1.33\left(\mathrm{~s}, 3 \mathrm{H}, \mathrm{CH}_{3}\right), 1.37\left(\mathrm{~s}, 3 \mathrm{H}, \mathrm{CH}_{3}\right), 1.79(\mathrm{~d}, J=8.0 \mathrm{~Hz}, 1 \mathrm{H}$, cyclopropane $\mathrm{H}), 2.18(\mathrm{t}, J=8.8 \mathrm{~Hz}, 1 \mathrm{H}$, cyclopropane $\mathrm{H})$, $2.33\left(\mathrm{~s}, 3 \mathrm{H}, \mathrm{CH}_{3}\right), 7.12(\mathrm{~d}, J=7.2 \mathrm{~Hz}, 1 \mathrm{H}, \mathrm{C}=\mathrm{CH}), 7.15-7.25$ (m, 4H, Ph), 7.34 (s, 1H, NH); Ms m/z (relative intensity/\%): $332\left(\mathrm{M}^{+}, 51\right), 296$ (31), 238 (29), 225 (10), 198 (21), 134 (100), 106 (83), 91 (55), 77 (22); anal. calcd. (\%) for $\mathrm{C}_{16} \mathrm{H}_{17} \mathrm{NOClF}_{3}$ : C 57.92, H 5.16, N 4.22, found (\%): C 58.09, H 5.11, N 4.15.
3-(2-Chloro-3,3,3-trifluoroprop-1-enyl)-2,2-dimethylN-(4-nitrophenyl)cyclopropanecarboxamide (4h): Yellow crystal; yield $72.6 \%$; m.p. $137-140{ }^{\circ} \mathrm{C} ;{ }^{1} \mathrm{H} \mathrm{NMR}\left(\mathrm{CDCl}_{3}\right) \delta$ : $1.36\left(\mathrm{~s}, 3 \mathrm{H}, \mathrm{CH}_{3}\right), 1.38$ (s, 3H, $\left.\mathrm{CH}_{3}\right), 1.86$ (d, $J=8.0 \mathrm{~Hz}, 1 \mathrm{H}$, cyclopropane $\mathrm{H}), 2.27(\mathrm{t}, J=8.8 \mathrm{~Hz}, 1 \mathrm{H}$, cyclopropane $\mathrm{H})$, $7.09(\mathrm{~d}, J=9.2 \mathrm{~Hz}, 1 \mathrm{H}, \mathrm{C}=\mathrm{CH}), 7.67-7.69(\mathrm{~m}, 2 \mathrm{H}, \mathrm{Ph}), 7.78(\mathrm{~s}$, $1 \mathrm{H}, \mathrm{NH}), 8.20-8.22(\mathrm{~m}, 2 \mathrm{H}, \mathrm{Ph}) ; \mathrm{Ms} \mathrm{m} / \mathrm{z}$ (relative intensity/ \%): 363 (M+, 70), 327 (20), 269 (23), 225 (32), 198 (74), 165 (100), 141 (90), 127 (19), 91 (29), 77 (15); anal. calcd. (\%) for $\mathrm{C}_{15} \mathrm{H}_{14} \mathrm{~N}_{2} \mathrm{O}_{3} \mathrm{ClF}_{3}$ : C 49.67, H 3.89, $\mathrm{N}$ 7.72, found (\%): C 49.83, H 3.87, N 7.75.

3-(2-Chloro-3,3,3-trifluoroprop-1-enyl)-2,2-dimethylN-(3-trifluoromethylphenyl)cyclopropanecarboxamide (4i): White crystal; yield 87.1 \%; m.p. $149-151^{\circ} \mathrm{C}$; ${ }^{1} \mathrm{H}$ NMR $\left(\mathrm{CDCl}_{3}\right) \delta:$ 1.34(s, 3H, $\left.\mathrm{CH}_{3}\right), 1.38\left(\mathrm{~s}, 3 \mathrm{H}, \mathrm{CH}_{3}\right), 1.82(\mathrm{~d}, J=$ $8.4 \mathrm{~Hz}, 1 \mathrm{H}$, cyclopropane $\mathrm{H}), 2.22(\mathrm{t}, J=8.8 \mathrm{~Hz}, 1 \mathrm{H}$, cyclopropane $\mathrm{H}), 7.13(\mathrm{~d}, J=9.2 \mathrm{~Hz}, 1 \mathrm{H}, \mathrm{C}=\mathrm{CH}), 7.34-7.68(\mathrm{~m}$, $4 \mathrm{H}, \mathrm{Ph}), 7.73(\mathrm{~s}, 1 \mathrm{H}, \mathrm{NH}) ; \mathrm{Ms} \mathrm{m} / \mathrm{z}$ (relative intensity/\%): 386 $\left(\mathrm{M}^{+}, 62\right), 350$ (19), 292 (31), 225 (20), 198 (59), 188 (100), 160 (49), 141 (45), 91 (13), 77 (12); anal. calcd. (\%) for $\mathrm{C}_{16} \mathrm{H}_{14} \mathrm{NOClF}_{6}$ : C 49.82, H 3.66, N 3.63, found (\%): C 49.89, H 3.69, N 3.66.

3-(2-Chloro-3,3,3-trifluoroprop-1-enyl)-2,2-dimethyl$\mathbf{N}$-(3,4-dichlorophenyl)cyclopropanecarboxamide (4j): White crystal; yield, $89.6 \%$; m.p. $133-134{ }^{\circ} \mathrm{C}$; ${ }^{1} \mathrm{H}$ NMR $\left(\mathrm{CDCl}_{3}\right) \delta: 1.32\left(\mathrm{~s}, 3 \mathrm{H}, \mathrm{CH}_{3}\right), 1.36\left(\mathrm{~s}, 3 \mathrm{H}, \mathrm{CH}_{3}\right), 1.79(\mathrm{~d}, J=$ $8.0 \mathrm{~Hz}, 1 \mathrm{H}$, cyclopropane $\mathrm{H}), 2.21(\mathrm{t}, J=8.8 \mathrm{~Hz}, 1 \mathrm{H}$, cyclopropane $\mathrm{H}), 7.10(\mathrm{~d}, J=9.2 \mathrm{~Hz}, 1 \mathrm{H}, \mathrm{C}=\mathrm{CH}), 7.23-7.25(\mathrm{~m}$, 1H, Ph), 7.32-7.35 (m, 1H, Ph), 7.45 (s, 1H, Ph), 7.70 (s, 1H, $\mathrm{NH}) ; \mathrm{Ms} \mathrm{m} / \mathrm{z}$ (relative intensity/\%): $385\left([\mathrm{M}-1]^{+}, 24\right), 350(26)$, 292 (37), 225 (32), 198 (65), 188 (100), 163 (31), 141 (24), 91 (8), 77 (6). Anal. calcd. (\%) for $\mathrm{C}_{15} \mathrm{H}_{13} \mathrm{NOCl}_{3} \mathrm{~F}_{3}$ : C 46.60, H 3.39, N 3.62, found (\%): C 46.81, H 3.42, N 3.65.

3-(2-Chloro-3,3,3-trifluoroprop-1-enyl)-2,2-dimethylN-(2,4-dichlorophenyl)cyclopropanecarboxamide (4k): White crystal; yield $87.5 \%$, m.p. $130-133{ }^{\circ} \mathrm{C} ;{ }^{1} \mathrm{H} \mathrm{NMR}\left(\mathrm{CDCl}_{3}\right)$ $\delta: 1.30\left(\mathrm{~s}, 3 \mathrm{H}, \mathrm{CH}_{3}\right), 1.37\left(\mathrm{~s}, 3 \mathrm{H}, \mathrm{CH}_{3}\right), 1.81(\mathrm{~d}, J=8.0 \mathrm{~Hz}$, $1 \mathrm{H}$, cyclopropane $\mathrm{H}), 2.23$ (t, $J=8.8 \mathrm{~Hz}, 1 \mathrm{H}$, cyclopropane $\mathrm{H}), 7.15(\mathrm{~d}, J=9.2 \mathrm{~Hz}, 1 \mathrm{H}, \mathrm{C}=\mathrm{CH}), 7.25-7.28(\mathrm{~m}, 2 \mathrm{H}, \mathrm{Ph})$, 7.39-7.42 (m, 1H, Ph), $7.82(\mathrm{~s}, 1 \mathrm{H}, \mathrm{NH}) ; \mathrm{Ms} \mathrm{m} / \mathrm{z}$ (relative intensity/\%): 385 ([M-1] $\left.]^{+}, 40\right), 350$ (13), 292 (39), 225 (41), 198 (73), 188 (100), 163 (29), 141 (24), 91 (9), 77 (12); anal. calcd. (\%) for $\mathrm{C}_{15} \mathrm{H}_{13} \mathrm{NOCl}_{3} \mathrm{~F}_{3}: \mathrm{C} 46.60, \mathrm{H} 3.39, \mathrm{~N} 3.62$, found (\%): C 46.76, H 3.33, N 3.68.

3-(2-Chloro-3,3,3-trifluoroprop-1-enyl)-2,2-dimethylN-(2,4-difluorophenylcyclopropanecarboxamide (41): White crystal; yield $84.2 \%$; m.p. $148-149{ }^{\circ} \mathrm{C} ;{ }^{1} \mathrm{H} \mathrm{NMR}\left(\mathrm{CDCl}_{3}\right)$

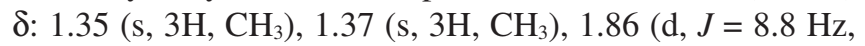
$1 \mathrm{H}$, cyclopropane $\mathrm{H}), 2.22(\mathrm{t}, J=8.8 \mathrm{~Hz}, 1 \mathrm{H}$, cyclopropane $\mathrm{H})$, 6.85-6.89 (m, 2H, Ph), 7.12 (d, J = 9.6 Hz, 1H, C=CH), 7.38 (s, 1H, NH), 8.17-8.21(m, 1H, Ph); Ms m/z (relative intensity/\%): $353\left(\mathrm{M}^{+}, 15\right), 318(16), 260(19), 225(10), 198(36)$, 163(48), 156(100), 141(29), 128(60), 91(10), 77(6); anal. calcd. (\%) for $\mathrm{C}_{15} \mathrm{H}_{13} \mathrm{NOClF}_{5}$ : C 50.93, H 3.70, N 3.96, found (\%): C 51.05, H 3.74, N 3.90.

\section{Biological activities}

Bioassay of fungicidal activities: The method for testing the primary biological activities was performed in an isolated 
culture. Under a sterile condition, $1 \mathrm{~mL}$ DMSO of title compound was added to the culture plates, followed by the addition of $9 \mathrm{~mL}$ of culture medium. The final mass concentration of the title compound was $50 \mu \mathrm{g} / \mathrm{mL}$. The blank assay was performed with $1 \mathrm{~mL}$ of sterile water. Circle mycelium with a diameter of $4 \mathrm{~mm}$ was cut using a drill. The culture plates were cultivated at $(24 \pm 1){ }^{\circ} \mathrm{C}$. The extended diameters of the circle mycelium were measured after $72 \mathrm{~h}$. The relative inhibition rate of the circle mycelium compared to blank assay was calculated via the following equation:

$$
\text { Relative inhibition rate }(\%)=\left[\frac{(\mathrm{CK}-\mathrm{PT})}{\mathrm{CK}}\right] \times 100 \%
$$

where $\mathrm{CK}$ is the extended diameter of the circle mycelium during the blank assay and PT, is the extended diameter of the circle mycelium during testing.

Bioassay of insecticidal activities: Insecticidal activities against Nilaparvata legen, Mythimna separate, Tetranychus cinnabarnus and Aphis medicagini were performed in the greenhouse. The bioassay was operated at $25 \pm 1^{\circ} \mathrm{C}$ according to statistical requirements. Assessments were made on a dead/ alive basis and mortality rates were corrected according to Abbott's formula. Per cent mortality was evaluated. Error of the experiments was $5 \%$. For comparative purpose, compound 4 were tested as control under the same conditions.

The insecticidal activities of compounds 4 were evaluated according FAO procedure. The insecticidal activity against oriental armyworm was tested by foliar application, individual corn leaves were placed on moistened pieces of filter paper in Petri dishes. The leaves were then sprayed with the test solution and allowed to dry. Then every 10 fourth-instar oriental armyworm larvae were put into each dish. Per cent mortalities were evaluated 2 days after treatment. Each treatment was replicated for three times.

\section{RESULTS AND DISCUSSION}

The synthetic routes of title compounds were illustrated as outlined in Scheme-I. The starting material $\lambda$-cyhalthrin acid (1) was treated with $\mathrm{SOCl}_{2}$ as chlorination reagent to generate acid chloride (2). The excess thionyl chloride was removed by reduced pressure distillation. For the next step the acyl chloride was used without additional purification. Then it reacted with substituted aniline at room temperature as shown in Scheme-I.

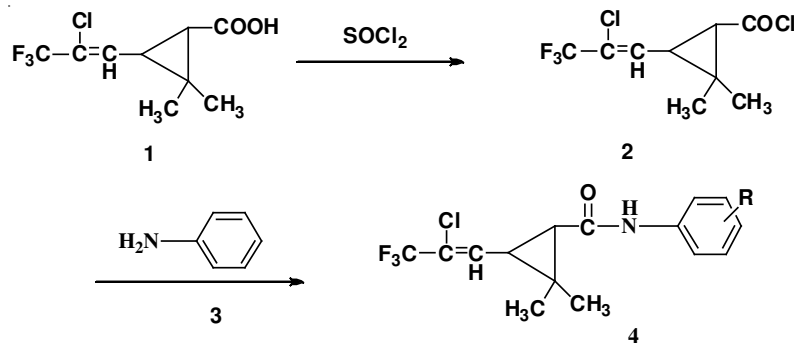

4a, R=H; 5b, R=4-Cl; 5c, R=2-Cl; 5d, R=3-Cl; 5e, R=4-Me; 5f, R=2-Me

$5 \mathrm{~g}, \mathrm{R}=3-\mathrm{Me} ; 5 \mathrm{~h}, \mathrm{R}=4-\mathrm{NO}_{2} ; 5 \mathrm{i}, \mathrm{R}=3-\mathrm{CF}_{3} ; 5 \mathrm{j}, \mathrm{R}=3,4-\mathrm{Cl}_{2} ; 5 \mathrm{k}, \mathrm{R}=2,4-\mathrm{Cl}_{2} ; 5 \mathrm{I}, \mathrm{R}=2,4-\mathrm{F}_{2}$ Scheme-I: Synthetic route of title compounds

The chemical structures of the title compounds were confirmed by ${ }^{1} \mathrm{H} \mathrm{NMR}$, mass and elemental analysis. The ${ }^{1} \mathrm{H}$
NMR, mass spectra and elemental analysis data of the compounds are in agreement with the proposed structures. In the ${ }^{1} \mathrm{H}$ NMR spectra, the $\mathrm{N}-\mathrm{H}$ protons of the amide derivatives were observed as singlets at 7.38-7.82 ppm. All other aromatic protons were observed in the expected regions. All the title compounds of mass spectra are molecular ion peak.

\section{Bioassay}

Fungicidal activity: The in vivo fungicidal results of all of the compounds against Rhizoctonia solani, Pseudoperonospora cubensis, Sphaerotheca fuliginea and Botrytis cinerea were listed in Table-1. As shown in Table-1, all these compounds did not display obvious fungicidal activities against Pseudoperonospora cubensis. Surprisingly, compound $4 \mathbf{k}$ exhibited good activity against Rhizoctonia solani $(63.8 \%)$. As shown in Table-1, compound displayed good activity against Botrytis cinerea. Among them, compounds $\mathbf{4 a}, \mathbf{4} \mathbf{c}, \mathbf{4 j}$ and $\mathbf{4 k}$ have good fungicidal activity (50-70\%) against Botrytis cinerea at the concentration of $200 \mu \mathrm{g} \mathrm{mL}^{-1}$. For the Sphaerotheca fuliginea, it was found that most of them had low activity.

\begin{tabular}{ccccc}
\multicolumn{5}{c}{ TABLE-1 } \\
\multicolumn{5}{c}{ FUNGICIDAL ACTIVITIES OF 4 (INHIBITION \%) } \\
\hline Compound & S. fuliginea & P. cubensis & B. cinerea & R. solani \\
\hline $\mathbf{4 a}$ & 26.3 & 0 & 54.2 & 0 \\
$\mathbf{4 b}$ & 18.6 & 0 & 20.3 & 18.9 \\
$\mathbf{4 c}$ & 16.3 & 0 & 50.7 & 0 \\
$\mathbf{4 d}$ & 14.2 & 0 & 29.6 & 0 \\
$\mathbf{4 e}$ & 8.8 & 5.6 & 38.9 & 15.7 \\
$\mathbf{4} \mathbf{f}$ & 7.6 & 0 & 10.6 & 0 \\
$\mathbf{4 g}$ & 7.2 & 0 & 9.8 & 0 \\
$\mathbf{4 h}$ & 0 & 0 & 6.8 & 0 \\
$\mathbf{4 i}$ & 0 & 0 & 20.8 & 0 \\
$\mathbf{4} \mathbf{j}$ & 30.5 & 0 & 71.8 & 0 \\
$\mathbf{4} \mathbf{k}$ & 38.9 & 16.9 & 57.8 & 63.8 \\
$\mathbf{4}$ & 0 & 3.8 & 8.8 & 10.2 \\
\hline
\end{tabular}

\section{ACKNOWLEDGEMENTS}

The project was supported by the Program of National Natural Science Foundation of China (B020706).

\section{REFERENCES}

1. (a) X.H. Liu, J.Q. Weng, C.X. Tan and H.J. Liu, Acta Cryst., E67, o1940 (2011); (b) X.H. Liu, J.Q. Weng, C.X. Tan, L. Pan, B.L. Wang and Z.M. Li, Asian J. Chem., 23, 4031 (2011); (c) H.J. Liu, J.Q. Weng, C.X. Tan and X.H. Liu, Acta Cryst., E67, o1940 (2011).

2. C.X. Tan, Y.X. Shi, J.Q. Weng, X. H. Liu, B.J. Li and W.G. Zhao, Lett. Drug Des. Discov., 9, 431 (2012).

3. (a) K. Matsuda, K. Iharada, K.H. Suzuki, M. Yamashita, H. Okimoto, K. Nishimura, K.T. Ueno and K. Komai, J. Pestic. Sci., 20, 487 (1995); (b) X.H. Liu, L. Pan, J.Q. Weng, C.X. Tan, Y.H. Li, B.L. Wang and Z.M. Li, Mol. Divers., 16, 251 (2012); (c) X.H. Liu, L. Pan, C.X. Tan, J.Q. Weng, B.L. Wang and Z.M. Li, Pestic. Biochem. Physiol., 101, 143 (2011).

4. (a) X.H. Liu, C.X. Tan and J.Q. Weng, Phosphorus Sulfur Silicon Rel. Elem., 186, 552 (2011); (b) P.Q. Chen, C.X. Tan, J.Q. Weng and X.H. Liu, Asian J. Chem., 24, 2808 (2012); (c) Y.L. Xue, Y.G. Zhang and X.H. Liu, Asian J. Chem., 24, 3016 (2012); (d) Y.L. Xue, Y.G. Zhang and X.H. Liu, Asian J. Chem., 24, 1571 (2012); (e) X.H. Liu, C.X. Tan and J.Q. Weng, Phosphorus Sulfur Silicon Rel. Elem., 186, 558 (2011); (f) X.F. Liu and X.H. Liu, Acta Cryst., E67, o202 (2011). 